BRAZILIAN JOURNAL

$\mathrm{OF}$

RADIATION SCIENCES

08-01A (2020) 01-16

\title{
Adaptação de um fantoma antropomórfico para representar um IOE no momento da administração de radiofármacos em medicina nuclear
}

\author{
Farias $^{a}$ W. A. W. A., Vieira ${ }^{a}$ J. W., Santana ${ }^{b}$ I. E., Silva ${ }^{b}$ V. H. F. F., \\ Lins $^{\mathrm{b}}$ J. A. G., Andrade ${ }^{\mathrm{c}}$ P. H. A., Lima ${ }^{\mathrm{b}, \mathrm{d}}$ F. R. A. \\ ${ }^{a}$ Instituto Federal de Educação, Ciência e Tecnologia de Pernambuco (IFPE Campus Recife) \\ 50740-540, Av. Prof. Luís Freire, 500, Recife-PE, Brasil. \\ ${ }^{b}$ Departamento de Energia Nuclear (DEN), Universidade Federal de Pernambuco (UFPE) \\ 50740-540, Av. Prof. Luís Freire, 1000, Recife-PE, Brasil. \\ ${ }^{c}$ Instituto Federal de Educação, Ciência e Tecnologia de Pernambuco (IFPE Campus Belo Jardim) \\ 55145-065, Belo Jardim-PE, Brasil \\ ${ }^{d}$ Centro Regional de Ciências Nucleares do Nordeste (CRCN-NE) \\ 50730-120, Av. Prof. Luís Freire, Recife-PE, Brasil. \\ Whoody_alem@hotmail.com
}




\section{RESUMO}

Modelos computacionais de exposição (MCEs) são usados em dosimetria numérica para estimar a dose absorvida, que corresponde à energia média depositada em um dado volume de massa, nos órgãos e tecidos radiossensíveis do corpo humano. São compostos, fundamentalmente, por fantomas computacionais, algoritmos simuladores de fontes radioativas e de um código Monte Carlo para simular o transporte e a interação da radiação com a matéria. Características do fantoma tais como anatomia, postura, massa corporal e estatura devem representar do modo mais realista possível a situação de exposição as radiações ionizantes para que os dados obtidos na simulação sejam mais fidedignos. Para tanto, os fantomas BREP (Boundary Representation) apresentam algumas vantagens quando comparados a outros tipos de fantomas, entre as quais a liberdade para modificações, além da precisão em representar a anatomia humana. Este trabalho teve por objetivo adaptar o fantoma BREP MARTIN (Male Adult with Macro Circulation and Lymphatic Vessels Phantom) para reproduzir de forma realista o momento em que o indivíduo ocupacionalmente exposto (IOE) faz a administração de radiofármacos (RF) via endovenosa em pacientes do setor de medicina nuclear (MN).

Palavras-chave: Dosimetria, Modelos computacionais de exposição, Boundary Representation.

\footnotetext{
ABSTRACT

Exposure computational models (MCEs) are used in numerical parameters to estimate dose absorption, which corresponds to the average energy deposited on a given mass volume in the radiosensitive organs and tissues of the human body. They are mainly composed by computational phantoms, simulative algorithms of radioactive sources and a Monte Carlo code for simulation or transport and interaction of pollution with matter. Ghost characteristics, such as anatomy, posture, body mass and height, should display the most realistic mode possible for the exposure situation as ionizing radiation for the data displayed in the most reliable simulations. Therefore, BREP (Border Representation) features have some advantages compared to other types of features, including freedom of change, and the accuracy of using the human anatomy. The aim of this study was to adapt the BREP MARTIN (male adult with macro circulation and phantom of lymphatic phantom) phantom to realistically reproduce or time when exposed busy individual (IOE) is intravenously administered radiopharmaceuticals (RF) to patients in the sector of nuclear medicine (MN).
}

Keywords: Dosimetry, Computer exposure models, Boundary representation. 


\section{INTRODUÇÃO}

A medicina nuclear (MN) consiste numa especialidade médica que utiliza fontes radioativas abertas (radiotraçadores ou radiofármacos) no diagnóstico e/ou tratamento de diversas patologias. A via de administração dessas substâncias pode ser oral, via respiratória ou injeção endovenosa, sendo esta última a mais utilizada.

Ao injetar o radiofármaco (RF), o indivíduo ocupacionalmente exposto (IOE) encontra-se numa situação de elevada exposição à radiação e é de extrema importância avaliar a distribuição de dose nos órgãos desse profissional quando ele realiza esse tipo de procedimento, visto que o mesmo repete essa operação várias vezes ao dia.

Todo IOE em MN que manipula e/ou administra RFs deve ser monitorado através de dosímetro individual de corpo inteiro, posicionado no tórax, além de dosímetro individual de extremidades [1]. A análise dos dosímetros é realizada mensalmente e fornece valores de dose efetiva. Contudo, a obtenção de tais dados é limitada a superfície do corpo, e não é possível saber com precisão a dose equivalente, distribuição de dose ou o quanto certo procedimento contribui na dose mensal desse trabalhador [2].

Para a obtenção de informações suplementares às obtidas pelos dosímetros podem ser utilizados modelos computacionais de exposição (MCEs). Entre outras aplicações, com os MCEs é possível otimizar procedimentos de radioproteção, simular situações de contaminação do ambiente pelas radiações ionizantes, ou ainda, obter a distribuição de dose para um dado procedimento realizado pelo IOE [3].

Um MCE é composto, fundamentalmente, por um fantoma computacional, um algoritmo simulador de uma fonte radioativa e um código Monte Carlo para simular o transporte e a interação da radiação com a matéria [2].

MCEs em que os parâmetros cinesiológicos do fantoma não correspondem com a postura real de exposição, podem gerar diferenças significativas nos resultados obtidos [4]. No entanto, a maioria dos MCEs são criados com fantomas em posição anatômica ou supina. Uma possível causa para a escassez de fantomas com posturas diferenciadas se deva a falta de flexibilidade dos 
fantomas de voxels, que durante algumas décadas, foi a única opção para simular o corpo humano com realismo anatômico.

Com advento dos fantomas Boundary Representation (BREP) é possível utilizar ferramentas computacionais para ajustar parâmetros antropométricos, anatômicos e cinesiológicos com relativa liberdade se comparados com fantomas de voxels [4]. Além disso, com essa nova geração de fantomas computacionais desenvolvidos pela comunidade científica, é possível criar fantomas completos sem o uso de imagens médicas, mantendo o realismo anatômico, a partir de imagens de referência [5].

Visando a construção de um MCE para realizar avaliações dosimétricas envolvendo profissionais de MN no momento da injeção de RFs em pacientes, o presente trabalho teve por objetivo adaptar o fantoma BREP, denominado MARTIN (Male Adult with Macro Circulation and Lymphatic Vessels Phantom), para representar esse trabalhador. Essas adaptações envolveram a mudança de postura, colocação de roupas, jaleco, sapatos, avental de chumbo e óculos plumbífero.

\section{MATERIAIS E MÉTODOS}

A primeira etapa deste trabalho foi desenvolvida no Centro de Medicina Nuclear de Pernambuco (CEMUPE), e consistiu na observação da rotina de trabalho dos IOEs. Foi constatado que na administração de RFs, na fossa antecubital dos pacientes, a maioria dos profissionais utilizava uma postura com o tronco flexionado e com o membro superior direito também flexionado na mesma direção como exemplificado na Figura 1. 


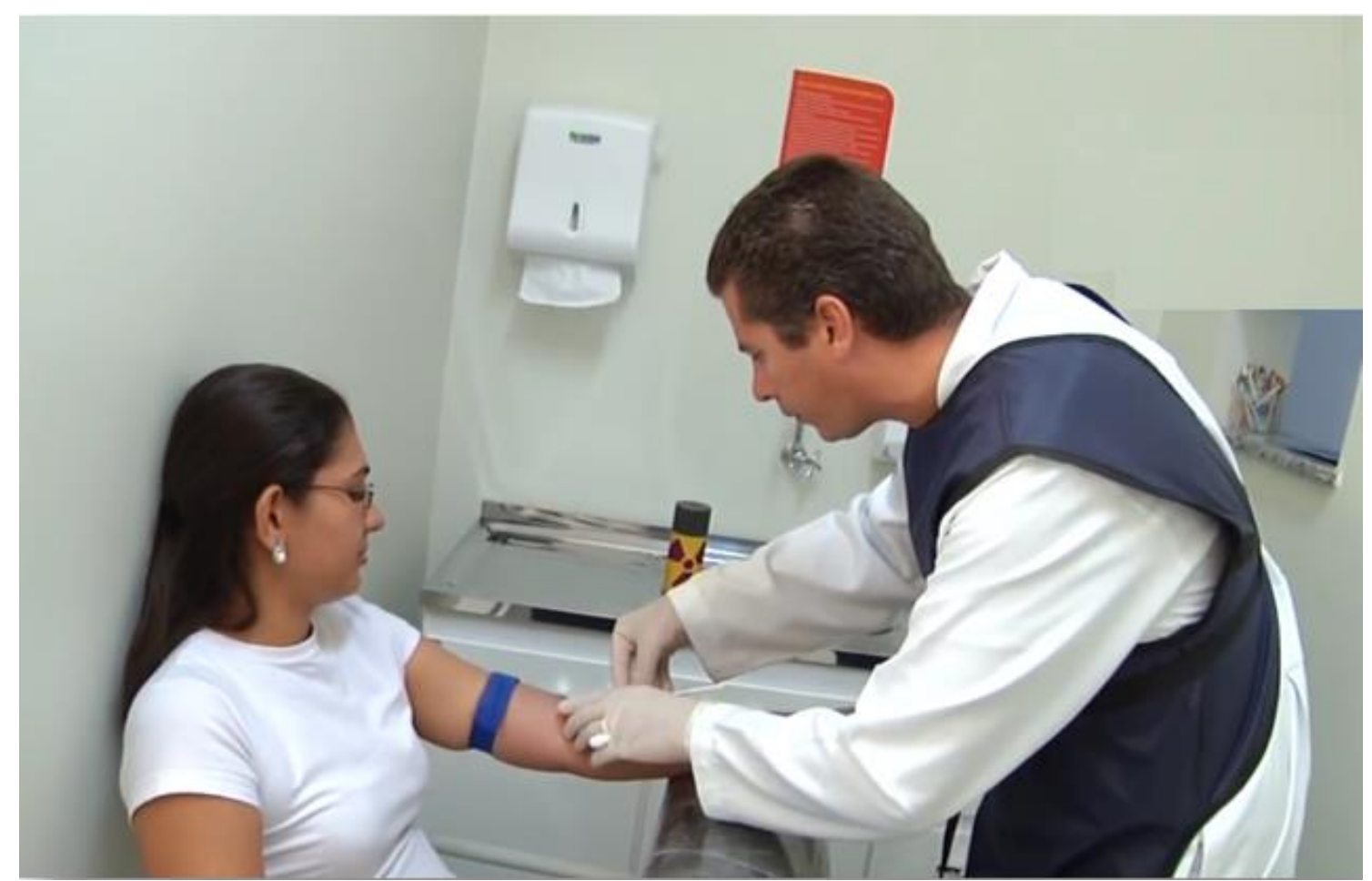

Figura 1: Postura usual para administração via endovenosa na fossa antecubital. Fonte: Núcleo Diagnóstico, 2012.

A parte computacional foi concebida no Laboratório de Dosimetria Numérica (LDN) do Instituto Federal de Educação, Ciência e Tecnologia de Pernambuco (IFPE), Campus Recife, em computadores que têm como principais itens de configuração um processador Intel Core i7 X990 @ 3,47GHz, 24 GB de RAM e sistema operacional Windows 7 Ultimate de 64 bits. Nestes computadores está disponível o fantoma MARTIN em formato .obj, além do Blender, versão 2.79 instalado.

Para a criação de um fantoma que representasse de forma satisfatória o IOE foi escolhido o MARTIN. Esse simulador antropomórfico é composto por uma coleção de 104 objetos de malhas poligonais, que descrevem seus órgãos e tecidos [5]. Desenvolvido pelo Grupo de Pesquisa em Dosimetria Computacional e Sistemas Embarcados (GPDC\&SE), o MARTIN representa um homem adulto jovem, despido, em posição ortostática com os membros superiores estendidos ao lado do tronco como pode ser visto na Figura 2. 


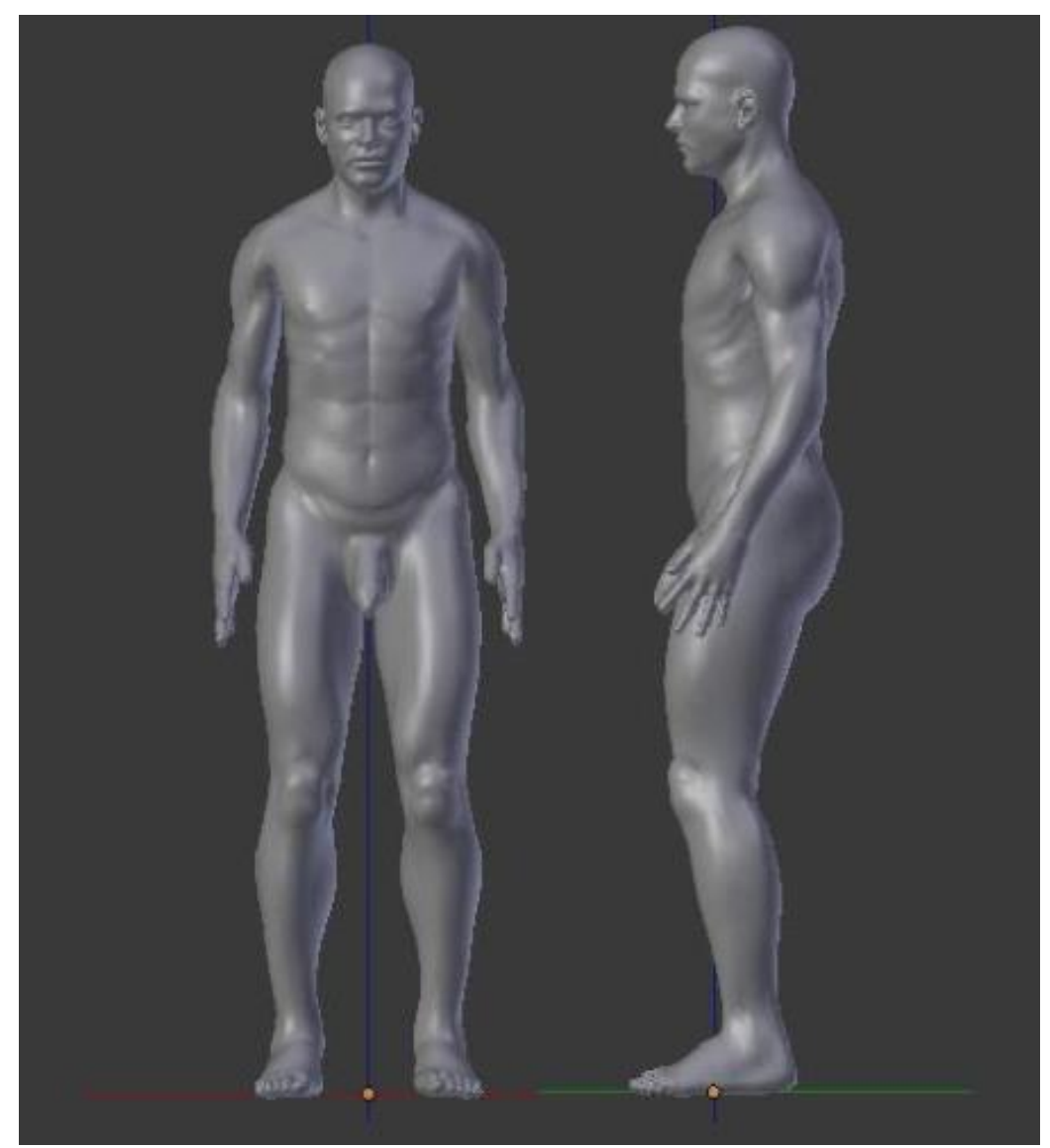

Figura 2: MARTIN.

O Blender foi escolhido para a realização desse trabalho, pois além de estar sob licença General Public License (GNU GPL) é um software de animação gráfica que oferece ferramentas robustas para animações e para a criação e edição de conteúdos em 3D. Com o Blender, ainda é possível adicionar ferramentas complementares (add-ons) proporcionado ainda mais funcionalidades ao software [6].

Pela natureza dos softwares de animação gráfica 3D, cujo a finalidade é a liberdade para criação de elementos gráficos, muitas vezes para a arte, não existe uma metodologia tida como correta para trabalhos executados nesses softwares [6]. Cabe ao usuário usar de sua expertise para escolher a melhor forma de realizar suas tarefas, e quais ferramentas utilizar no processo. A seguir, serão descritas as ferramentas e a metodologia usadas nesse trabalho, para adaptar o MARTIN para representar o IOE em MN. 


\subsection{Rigging e Skinning}

No ambiente do Blender, as alterações no MARTIN iniciaram com o processo de rigging, que consiste em equipar personagens (aqui referenciado como fantoma ou MARTIN) com estruturas de controle para animações, denominadas como bones. Como outros objetos do Blender os bones, que são objetos do modificador $^{l}$ armação, também possuem ponto de origem, posição, rotação e um fator de escala. Tais condições devem ser ajustadas, sempre que preciso, para que a armação funcione de forma satisfatória.

Como pode ser visto na Figura 3 os bones são compostos de raiz, corpo e ponta. O primeiro é o local de rotação do bone e, deve ser posicionado nos pontos de articulações do fantoma para simular as articulações do corpo humano. A ponta é o ponto de extrusão para criação de uma armação contínua e hierarquizada, ou seja, o primeiro bone criado será o "pai” do bone criado por extrusão a partir dele e assim sucessivamente. Com isso, ao movimentar o superior hierárquico de uma cadeia, todos os bones inferiores hierarquicamente, seguirão o movimento do "pai”, mas não o contrário.

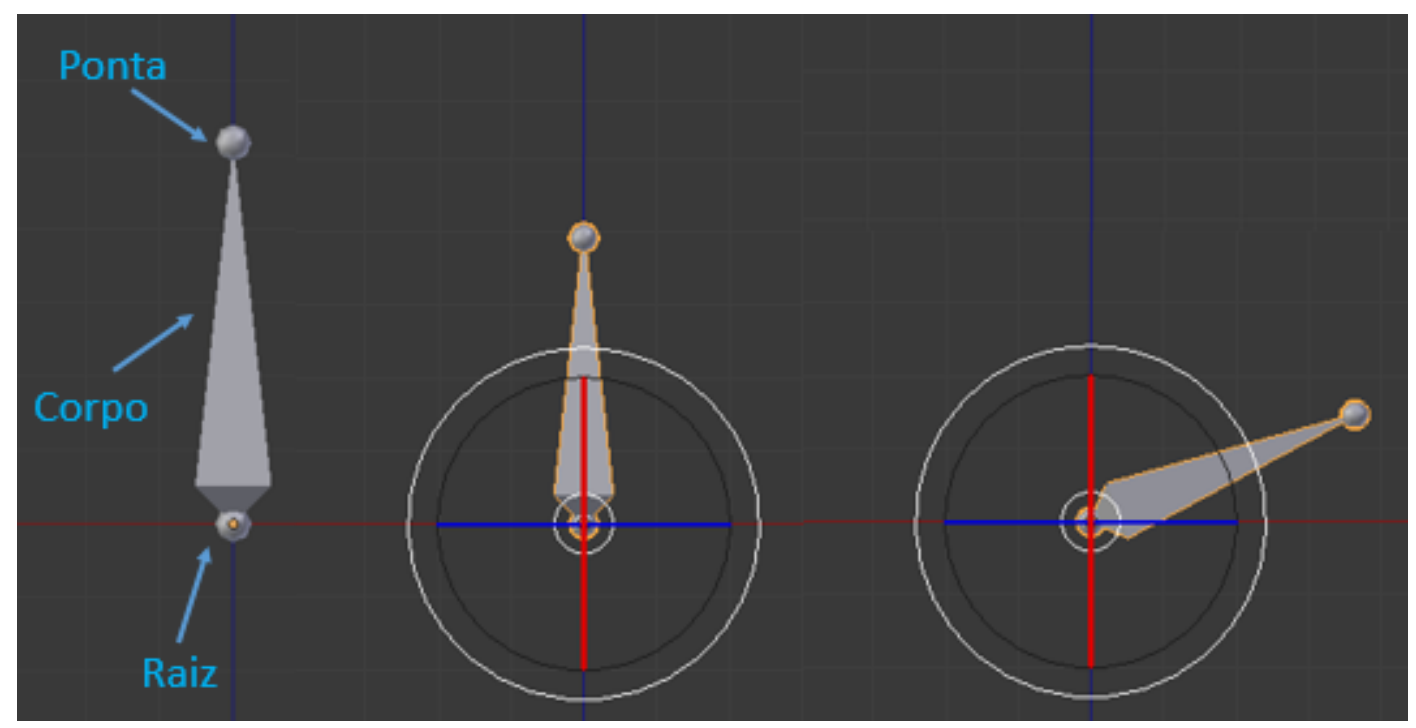

Figura 3: Bone.

Para a criação do rigging no MARTIN optou-se por criar bones em todo o fantoma de modo a reproduzir de forma simplificada o esqueleto humano. Com isso, foram criados bones até mesmo

\footnotetext{
${ }^{1}$ Modificadores são automatizações que auxiliam o usuário na realização de tarefas, especialmente, quando estas são complexas ou repetitivas [6].
} 
para os membros inferiores e para outras estruturas que não passariam por alteração de postura, isto é, não sofreriam mudanças de localização no plano tridimensional do Blender. Isso foi feito para que vértices mais afastados das regiões a serem movimentadas (parte superior do corpo) fossem ligados aos bones criados em sua proximidade, para minimizar que vértices das partes fora da região de movimentação fossem atraídos por bones das regiões de interesse.

Para reproduzir de forma mais realista possível o sistema locomotor do corpo humano a raiz dos bones, que são os pontos de rotação, foram inseridos nos locais de articulação do MARTIN como pode ser visto na Figura 4. Ao término do rigging foram criados 61 bones ao longo de todo corpo do fantoma.

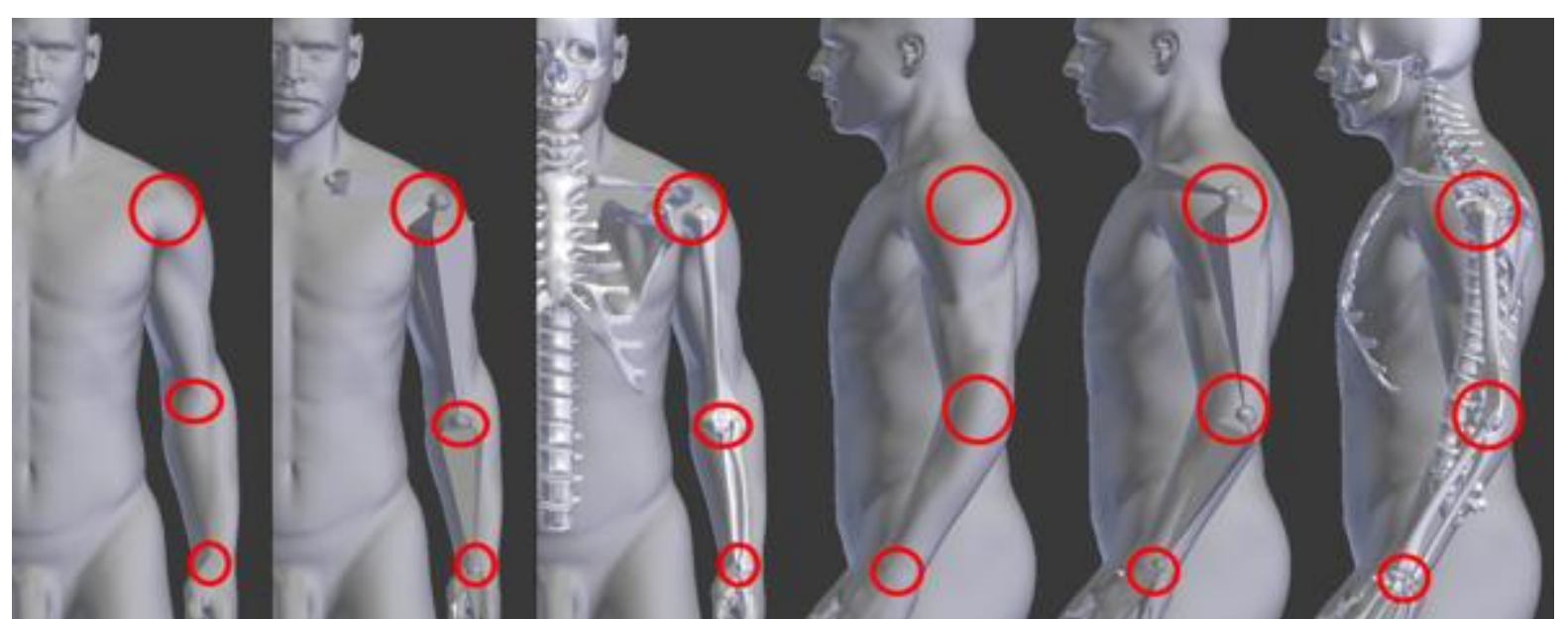

Figura 4: Projeções frontais e em perfil, respectivamente, mostrando alguns pontos de articulação do membro superior esquerdo e, onde estão inseridas às raízes dos bones.

Para que a malha poligonal seja movimentada pela armação é preciso, ainda, realizar o processo de skinning. Esse processo consiste em conectar a armação à malha poligonal para que esta possa ser deformada pela ação do usuário sobre os controles da malha.

No Blender é possível fazer o skinning por diversas formas. Nesse trabalho optou-se por atribuir o modificador armadura a toda a malha poligonal, através da opção pesos de influência automáticos. Esse método é mais preciso que os outros disponíveis no software, entretanto, sua configuração é mais complexa e demorada [6]. Com o uso dessa opção, a armação torna-se "pai" (superior hierárquico) de todos os 104 objetos do MARTIN. Com isso, o algoritmo do Blender 
calcula para cada bone a região de atuação do controle e sua influência sobre a área, baseando-se na proximidade entre os bones e os vértices de cada objeto. Contudo, após o término do skinning é necessário que o usuário realize modificações nos pesos atribuídos automaticamente para que a armação funcione corretamente, como será mostrado no item 2.3.

\subsection{Alteração de parâmetros cinesiológicos (animação)}

Uma vez que a malha poligonal é deformável pelo modificador armação, é possível iniciar a animação dos objetos vinculados a ele. A animação consiste em alterar a posição de descanso da armação para poses desejadas pelo usuário, variando com o tempo ou em poses estáticas. Para tanto, o Blender dispõe do modo pose. Com ele é possível realizar movimentos e rotações na armação que deformam a malha poligonal.

A primeira modificação desta etapa consistiu em rotacionar no eixo $\mathrm{x}$, do sistema de coordenadas do Blender, uma série de bones criados para reproduzir os movimentos da coluna vertebral. Os bones citados controlam os movimentos do tronco, cabeça e pescoço. Desta forma, ao realizar uma flexão da coluna vertebral as demais estruturas controladas por esses bones seguiram o movimento.

A rotação aplicada a cada bone foi baseada na deformação gerada no objeto pele. A Figura 1 foi usada como referencial para as modificações. Com isso, quando a pele assumiu um grau de flexão similar à da imagem de referência a rotação desses bones foi encerrada.

Para o membro superior direito a metodologia aplicada foi análoga à usada para simular os movimentos da coluna vertebral, descrita anteriormente. Desta forma, O bone situado na altura do braço, posicionado para simular os movimentos do úmero, foi rotacionado no eixo x. Por este bone ser o pai dos demais ossos do membro superior direito, após a rotação realizada, seus filhos seguiram o movimento.

\subsection{Pintura de influência}

O skinning, executado por pesos de influência automáticos proporciona ao usuário do Blender a automatização dessa tarefa. Entretanto, como já esperado, ocorreram várias sobreposições de influência, isto é, grupos de vértices que não deveriam deformar por ação de bones distantes, 
acabaram sofrendo influência desses controles.

Para contornar tal limitação de seu algoritmo o Blender dispõe do modo pintura de influência. Com ele é possível pintar manualmente os pesos gerados de forma automática pelo software. A Figura 5 mostra o espectro de cores usado pelo Blender para controlar os pesos de influência atribuídos aos bones na malha poligonal. Sendo, a cor azul (frio), pouca influência, e valores mais quentes próximos do 1.0 (alta influência).

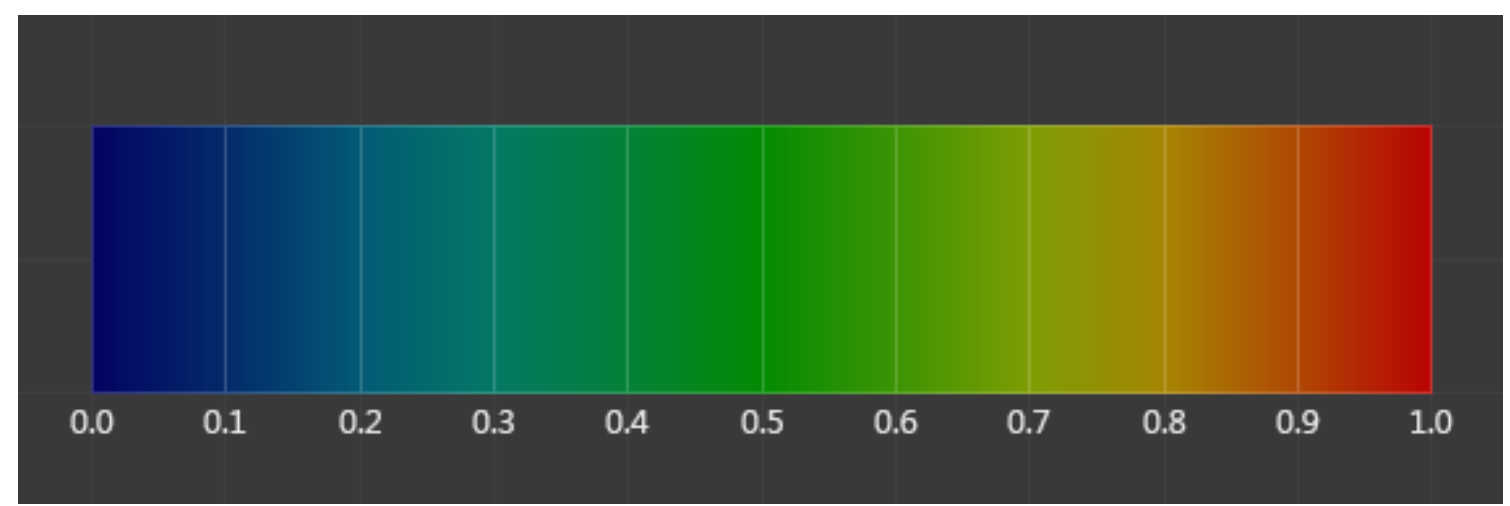

Figura 5: espectro de pesos de influência.

Fonte: Blender, 2017.

No modo pintura de influência as ferramentas mais usadas para a correção dos pesos gerados no skinning foram as de adição e a de subtração. A primeira permite tornar uma região mais quente. Por sua vez, a ferramenta de subtração permite ao usuário diminuir a influência que um bone gera na malha. Na Figura 6 pode ser visto a aplicação da ferramenta de subtração tornando algumas estruturas internas mais frias aos bones do membro superior direito. 


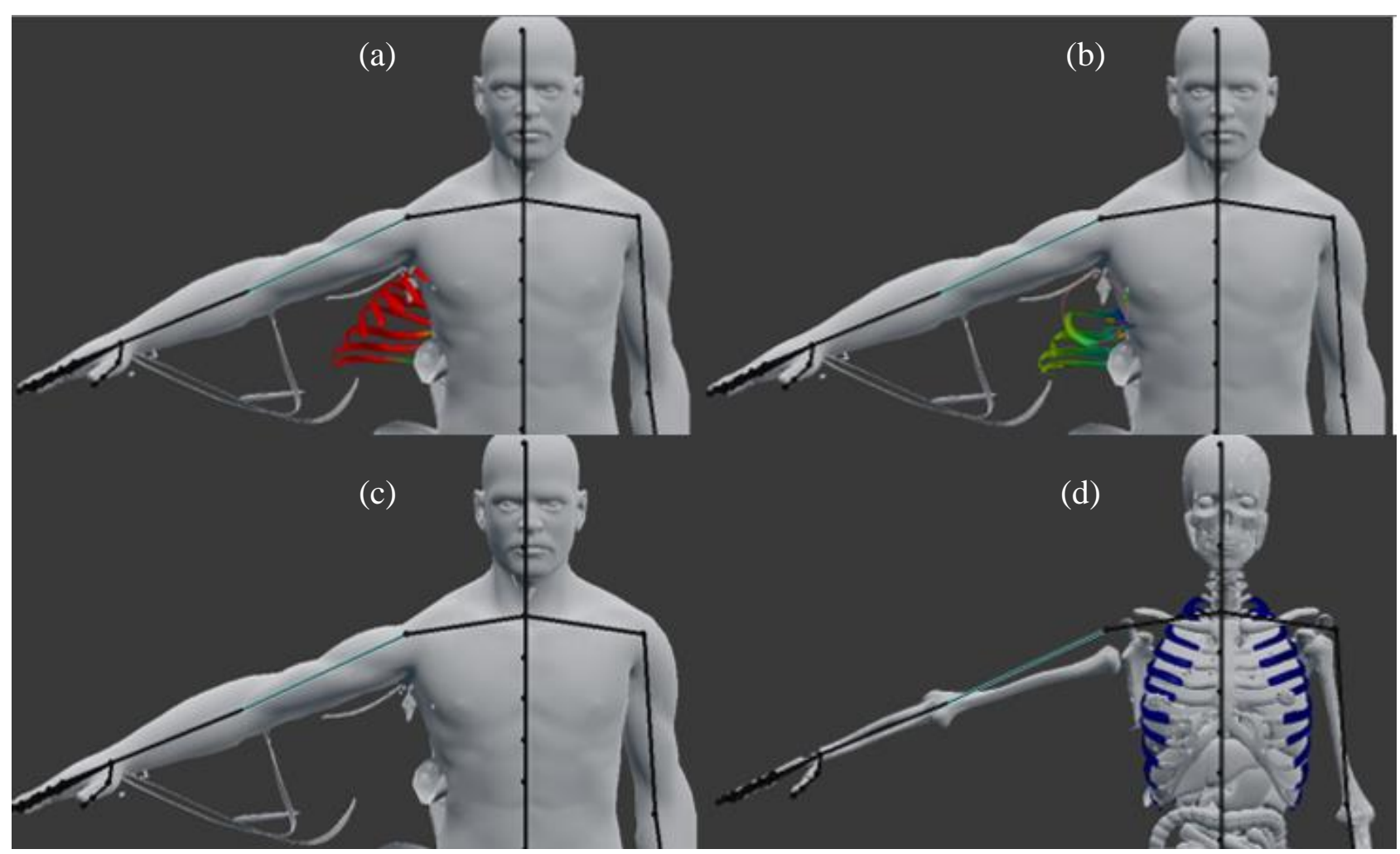

Figura 6: Correção dos pesos de influência gerados no skinning. Costelas com regiões quentes sendo influenciadas pelo bone controlador do braço (a), processo de subtração de influência (b), pesos de influência da costela corrigidos com pele (c) e sem pele (d), respectivamente.

O processo mostrado na Figura 6 foi executado para todos os objetos do MARTIN. O esqueleto foi o primeiro conjunto de objetos que passaram pela correção de pesos de influência, pois o mesmo serviu como referencial para pintura de influência dos demais objetos fornecendo para eles marcos anatômicos. Desta forma, uma por uma as estruturas tiveram a sua localização anatômica checada, com o auxílio de um atlas anatômico [7]. Esta foi a etapa mais demorada na realização desse trabalho, pois exige do usuário domínio da ferramenta, conhecimentos em anatomia, cautela e uma destreza quase artística para obtenção de um resultado satisfatório.

\subsection{Modelagem 3D}

A modelagem tridimensional ou simplesmente modelagem 3D tem como objetivo representar objetos tridimensionais através de uma representação matemática. Para tanto, objetos primitivos 
como cubos, círculos, cilindros, planos entre outros, são editados para representar objetos mais complexos.

Para a criação do avental de chumbo, sapatos e óculos plumbífero o objeto plano foi usado como base. Foram usadas a ferramenta de extrusão, que duplica os vértices e cria arestas e faces mantendo a conexão com os vértices iniciais para criar um objeto único, além das ferramentas de rotação, escalonamento e edição proporcional. Desta forma, a partir de uma malha primitiva 2D com uma única face quádrupla composta por 4 vértices foram obtidos tais objetos (Figuras 7 e 8 ).

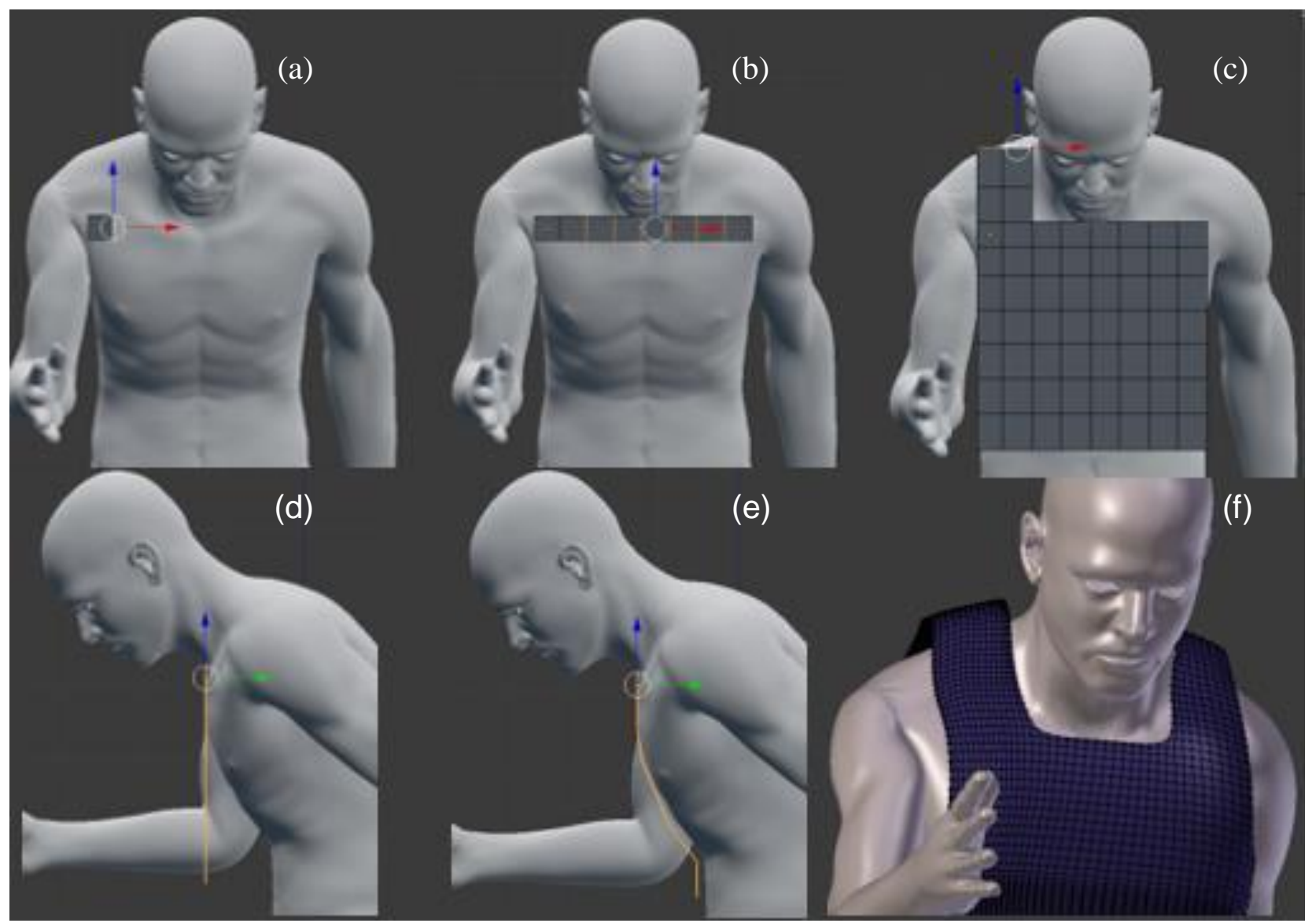

Figura 7: modelagem do avental de chumbo. (a) objeto base, (b) extrusão no eixo $x$, (c) criação de faces sobre o ombro direito e da parte posterior, por extrusão, $(d)$ visão em perfil mostrando que o avental não foi criado rente ao corpo, (e) uso de movimentações e edição proporcional para deixar o objeto rente ao corpo, $(f)$ avental de chumbo finalizado. 


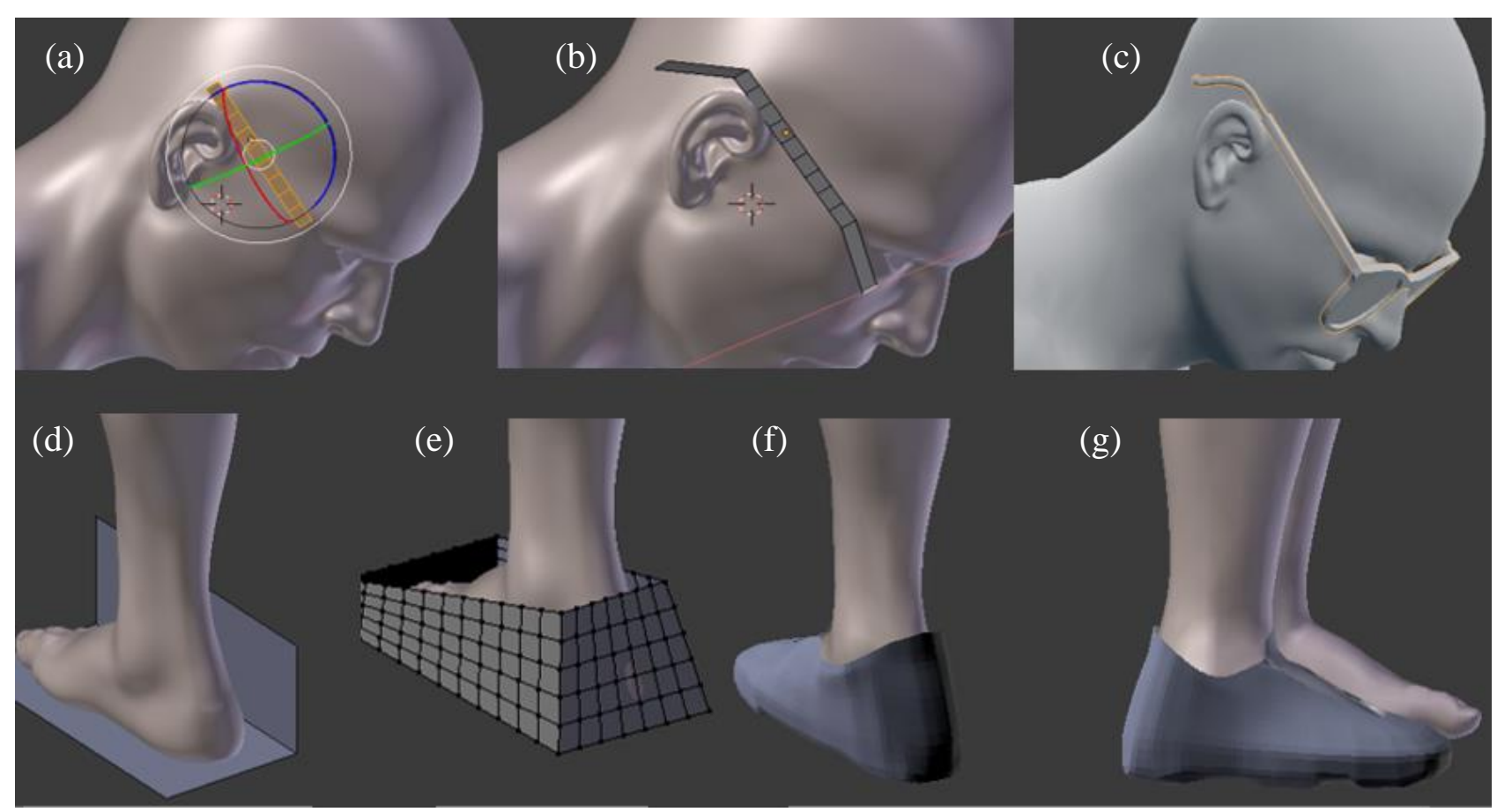

Figura 8: criação do objeto óculos. (a) rotação dos polígonos para deixá-los rente ao objeto pele, (b) movimento ao longo do eixo x para posicionamento da haste do objeto óculos, (c) óculos finalizados. Criação do objeto sapatos. (d) malha após a extrusão do objeto base ao longo do eixo $z,(e)$ malha poligonal após extrusões, movimentações e ajuste de escala, $(f)$ e $(g)$ objeto finalizado nas vistas oblíqua posterior e em perfil, respectivamente.

Para gerar o protetor de tireoide a metodologia usada foi análoga à empregada para a criação dos objetos já citados. Contudo, o objeto base para criação do protetor de tireoide foi o cilindro, como pode ser visto na Figura 9.

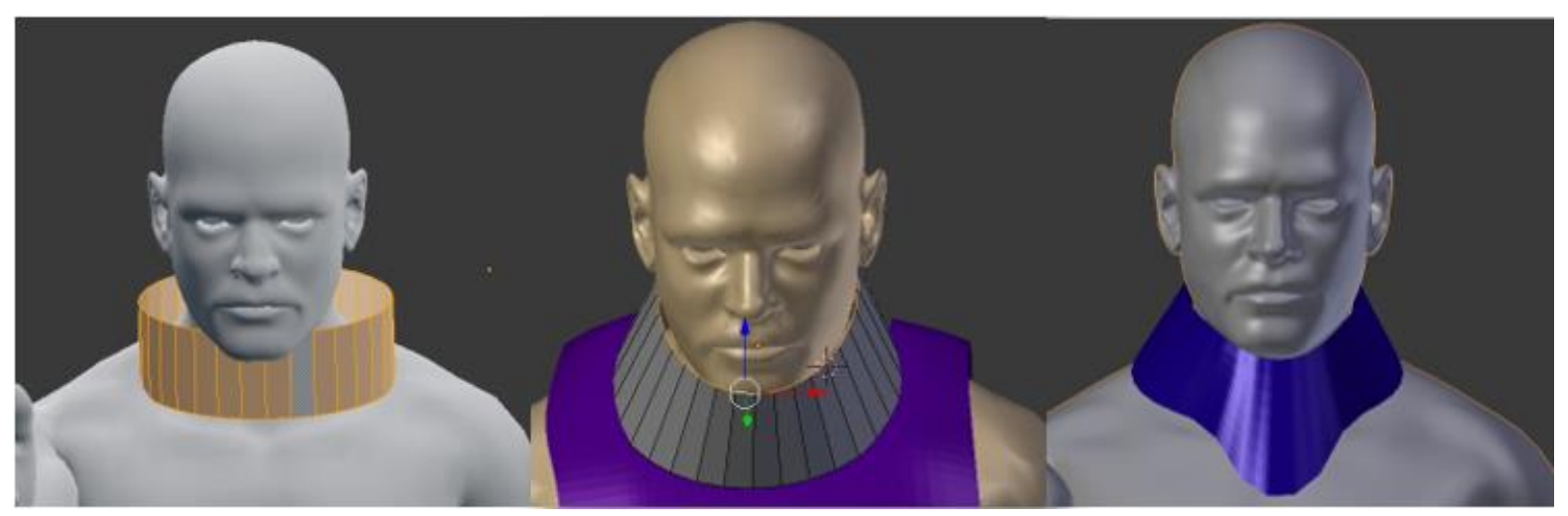

Figura 9: modelagem do protetor de tireoide. 
$\mathrm{Na}$ criação das roupas e do jaleco à malha poligonal do objeto pele, foi usada como base para produção dessas estruturas. Para tanto, as faces das áreas de interesse foram selecionadas no mesmo formato dos objetos que seriam criados, duplicados e tiveram suas escalas aumentadas. Com isso, foi gerada uma nova "pele" no formato selecionado previamente. Ajustes foram feitos na nova "pele", com as ferramentas já citadas, e como pode ser visto na Figura 10 a técnica empregada para criação desses objetos se mostrou satisfatória para criação da camisa e dos demais objetos.

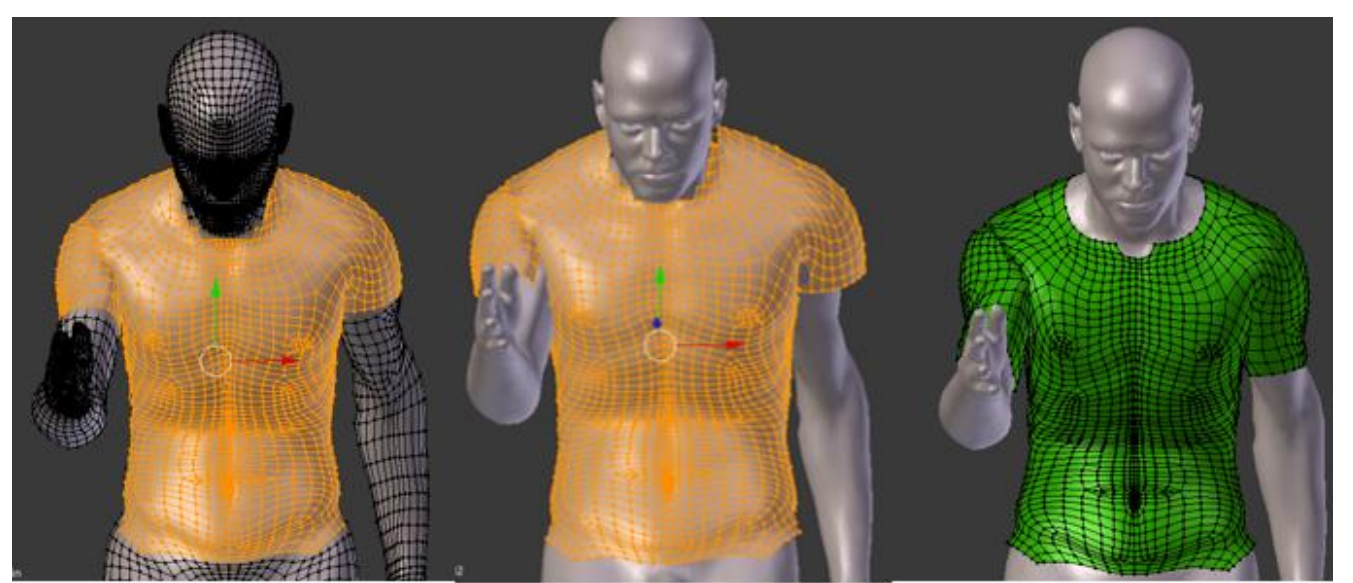

Figura 10: Criação do objeto camisa. Seleção das faces (a), aumento da escola (b), camisa finalizada (c).

\section{RESULTADOS E DISCUSSÃO}

Através da metodologia descrita, foi possível obter um fantoma de malhas poligonais com postura, vestimentas e EPIs adaptados, que representa de forma semelhante o IOE no momento da injeção de RFs em MN como pode ser visto na Figura 11. 


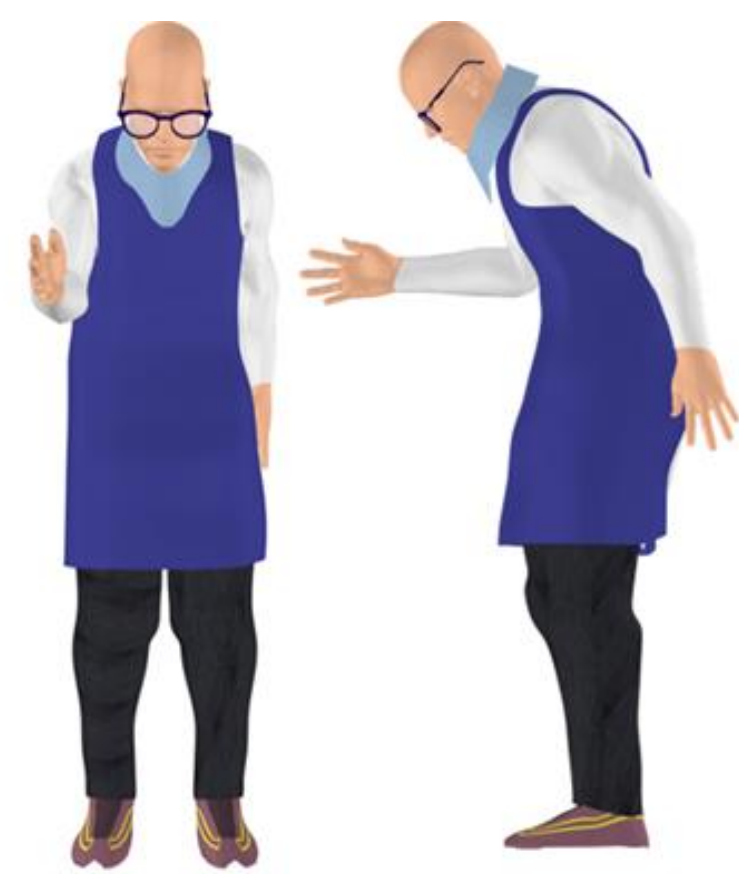

Figura 11: MARTIN após as modificações em projeção frontal e em perfil esquerdo.

O processo empregado para animação do fantoma, bem como, a criação de roupas e EPIs evidencia as principais vantagens dos fantomas BREP, que é a liberdade de criação de estruturas e a flexibilidade desse tipo de simulador antropomórfico.

\section{CONCLUSÕES}

O conjunto de modificações empregadas nesse trabalho, como a adaptação da postura e a criação de roupas e EPIs, resultou em um fantoma de alta especificidade para proteção radiológica do IOE em MN.

Este trabalho apresenta uma metodologia para proporcionar ainda mais robustez aos fantomas BREP, pois além da liberdade de criação dos objetos gráficos, característica deste tipo de fantoma, o método de animação utilizado proporciona a concepção de simuladores mais realistas.

A descrição dos processos usados para a animação do fantoma busca complementar os dados, escassos na literatura, sobre os meios de desenvolvimento de objetos gráficos e animação desses objetos aplicados a dosimetria numérica. 
Espera-se utilizar o fantoma adaptado em um MCE para avaliar a influência da administração do RF na dose mensal do trabalhador.

\section{AGRADECIMENTOS}

O presente trabalho foi realizado com apoio do Instituto Federal de Educação, Ciência e Tecnologia de Pernambuco (IFPE).

\section{REFERÊNCIAS}

[1] COMISSÃO NACIONAL DE ENERGIA NUCLEAR. CNEN-NN 3.05 - Requisitos de Radioproteção e Segurança para Serviços de Medicina Nuclear. Brasil, 1996.

[2] VIEIRA, J. W. Construção de um Modelo Computacional de exposição para cálculos dosimétricos utilizando o código Monte Carlo EGS4 e fantomas de voxels. Tese (Doutorado em Tecnologias Energéticas e Nucleares) - Departamento de energia nuclear, Universidade Federal de Pernambuco, Recife, 2004.

[3] VIEIRA, J. W. et al. Desenvolvimento de algoritmos simuladores de fontes radioativas planares para uso em modelos computacionais de exposição. Brazilian Journal Of Radiation Sciences , v. 1, p. 01-17, 2013.

[4] LOMBARDO P. A. et al. Developmentand validation of the realistic anthropomorphic flexible (raf) phantom. Health Physics, v. 114, n. 5, p. 486-499, 2018.

[5] ANDRADE, P. H. A. Construção e voxelização de um fantoma mesh masculino adulto com macro circulação e vasos linfáticos. 2018. Tese (Doutorado em Tecnologias Energéticas e Nucleares) - Departamento de energia nuclear, Universidade Federal de Pernambuco, Recife, 2018.

[6] BLENDER FOUNDATION. Manual de referência do blender: versão 2.79: Disponível em: https://docs.blender.org/. Acesso em: 01 ago. 2018.

[7] NETTER, Frank Henry. Atlas de anatomia humana. 6. ed. Rio de Janeiro: Elsevier, 2015. 